\title{
Evaluation of two methods of in vitro production of ovine embryos using fresh or cryopreserved semen
}

\author{
R. Romão ${ }^{\mathrm{a}, *}$, C.C. Marques ${ }^{\mathrm{b}}$, M.C. Baptista ${ }^{\mathrm{b}}$, M.I. Vasques $^{\mathrm{b}}$, J.P. Barbas $^{\mathrm{b}}$, A.E.M. Horta ${ }^{\mathrm{b}}$, \\ N. Carolino ${ }^{b, d}$, E. Bettencourt ${ }^{\mathrm{a}}$, C. Plancha ${ }^{\mathrm{c}}$, P. Rodrigues ${ }^{\mathrm{c}}$, R.M. Pereira ${ }^{\mathrm{b}, \mathrm{d}}$ \\ a Escola de Ciências e Tecnologia, Universidade de Évora, Polo da Mitra, Apartado 94, 7002-554 Évora, Portugal \\ b Unidade de Recursos Genéticos, Reprodução e Melhoramento Animal, INRB IP, L INIA-Santarém, Quinta da Fonte Boa, 2005-048 Vale de Santarém, Portugal \\ c Instituto de Histologia e Biologia do Desenvolvimento, Faculdade de Medicina da Universidade de Lisboa, Av. Professor Egas Moniz, 1649-028 Lisboa, \\ Portugal \\ ' Escola Universitária Vasco da Gama, Mosteiro de S. Jorge de Milréu, Estrada da Conraria, 3040-714 Castelo Viegas, Coimbra, Portugal
}

\section{A R T I C L E I N F O}

\section{Article history:}

Received 11 January 2012

Received in revised form 30 July 2012

Accepted 30 July 2012

Available online 18 August 2012

\section{Keywords:}

Ovine

Fresh semen

Frozen semen

Oocyte maturation

In vitro embryos

Cryopreservation

\begin{abstract}
A B S T R A C T
Successful production of high quality blastocysts depends on the use of a culture system that ensures the acquisition of developmental competence by the maturing oocyte followed by an efficient in vitro fertilization. In the present work the effect of FSH and pyruvate in an EGF containing medium for ovine oocyte maturation prior to insemination with fresh $(F)$ or frozen-thawed (FT) semen on embryo developmental competence and cryosurvival was determined. Sheep oocytes were matured in two culture media (M1 and M2, respectively; $\mathrm{M} 1=\mathrm{CM}+\mathrm{EGF}, n=836$ and $\mathrm{M} 2=\mathrm{CM}+\mathrm{EGF}+$ pyruvate $+\mathrm{FSH}, n=850)$ for $22 \mathrm{~h}$ and then fertil ized using FT or F spermatozoa $(\mathrm{M} 1 \times \mathrm{FT}=371, \mathrm{M} 2 \times \mathrm{FT}=359, \mathrm{M} 1 \times \mathrm{F}=353$ and $\mathrm{M} 2 \times \mathrm{F}=372$, 9 replicates) from Merino rams $(n=3)$. After embryo culture and evaluation, good quality blastocysts (grade 1) were vitrified in OPS. Post-thawed embryo integrity, re-expansion and number of total and viable cells were assessed. Oocyte maturation rates presented no differences $(P>0.05)$ between treatments $(M 1=87.0 \pm 4.1$ and $\mathrm{M} 2=86.7 \pm 3.9 \%)$ as well as embryo developmental rates either for maturation media or semen status. However, fresh semen improved blastocyst quality (grade 1 embryos $\mathrm{F}=52.5 \pm 4.8 \%$ and $\mathrm{FT}=39.0 \pm 4.4 \%$, $P=0.01$ ). Grade 1 blastocysts presented similar post-thawed integrity and re-expansion rates. After $3 \mathrm{~h}$ of culture, expansion rates were higher $(P=0.05)$ for $\mathrm{M} 2 \times \mathrm{F}$ warmed embryos $(80.0 \pm 8.3 \%)$ than for $\mathrm{M} 1 \times \mathrm{F}(54.3 \pm 10.4 \%)$. Results seem to confirm the existence of a synergistic effect between FSH, EGF and pyruvate upon cytoplasmic maturation of ovine oocytes. Moreover, in vitro fertilization by fresh semen clearly improves ovine embryo developmental competence by enhancing morphological blastocyst quality. The beneficial effect of M2 on cryosurvival was only observed in embryos derived from fresh semen. Therefore these combined strategies enhance embryo cryosurvival.
\end{abstract}

(C) 2012 Elsevier B.V. All rights reserved.

\footnotetext{
* Corresponding author. Tel.: +351 266760800x24598; fax: +351266760963.

E-mail addresses: rjromao@uevora.pt (R. Romão), nvarandamarques@gmail.com (C.C. Marques),baptista.sao@gmail.com (M.C. Baptista), irene.vasques@gmail.com (M.I. Vasques), jpbarbas@gmail.com (J.P. Barbas), horta.antonio@mail.telepac.pt (A.E.M. Horta), carolinonuno@hotmail.com (N. Carolino), emvb@uevora.pt (E. Bettencourt), cplancha@fm.ul.pt (C. Plancha), patyrodrigues@yahoo.com (P. Rodrigues), rosalnp@gmail.com (R.M. Pereira).
}

\section{Introduction}

Embryo production in small ruminants is a difficult task demanding experience and expensive facilities. The relative inefficiency of in vivo embryo production limits its use in sheep (Thibier and Guérin, 2000). In spite of the efforts towards the improvement of in vitro techniques by collecting oocytes in slaughterhouses or systematically pick them up from live animals, embryo production rates are 
still far from ideal, and transferable blastocysts are inferior in quality and cryotolerance compared to their in vivo counterparts (Papadopoulos et al., 2002; Cognié et al., 2003).

Several oocyte maturation protocols have been developed for in vitro fertilization (IVF) either with fresh or frozen-thawed semen (Dattena et al., 2004; Pereira et al., 2009). Regarding oocyte maturation, differences exist in the developmental competence of those matured in vivo compared to the in vitro ones (van de Leemput et al., 1999). Furthermore oocytes that have been exposed to a gonadotropin stimulus either in vivo or during in vitro culture are more competent to support embryonic development following fertilization (Rizos et al., 2002; Farin et al., 2007). The presence of receptors for growth factors such as insulin-like growth factor-1(IGF-1) and epidermal growth factor (EGF) in granulosa cells and oocytes suggest their involvement in the maturation process and developmental competence (Feng et al., 1987; Wang et al., 2009; Procházka et al., 2011) at least in early stages (Kelly et al., 2008). However, completion of meiosis in ovine oocytes and embryo development were significantly stimulated by the presence of FSH or EGF in the maturation medium, whereas IGF-1 was not effective (Guler et al., 2000). Recently a synergistic effect of FSH and EGF on cytoplasmic maturation of porcine oocytes was reported by Uhm et al. (2010). Thus, it is possible that FSH and EGF work in concert to regulate oocyte maturation and optimize oocyte developmental competence in vitro. During oocyte maturation, FSH and EGF promote glucose metabolism in cumulus cells through the hexosamine biosynthetic pathway (HBP) leading to cumulus expansion by the synthesis of extracellular matrices (Buccione et al., 1990). On the other hand, the oocyte has a poor capacity to use glucose which must be metabolized to pyruvate by the cumulus cells to supply the oocyte. Sugiura et al. (2005) suggest that FSH/EGF stimulation of HBP during in vitro maturation denies the oocyte-mediated promotion of glycolysis that was seen within the cumulus cells resulting in an energy imbalance in the oocyte. Pyruvate supplementation should minimize this fact.

Moreover, reports comparing IVP embryos competence and cryotolerance after IVF using fresh or cryopreserved sperm are sparse. Although cryopreserved ram semen is easily available for routine use, freezing and thawing disrupts the stability of ram sperm chromatin, most likely reducing the fertilization efficiency of cryopreserved semen in vitro and in vivo (Peris et al., 2004). Consequently the use of fresh semen could improve embryo production and cryopreservation results. The purpose of this study was to determine the effect(s) of the inclusion of FSH and pyruvate in an EGF containing maturation medium as well as the use of fresh and frozen-thawed sperm for fertilization on ovine embryo developmental competence and cryosurvival.

\section{Materials and methods}

All chemicals used were purchased from Sigma Aldrich Chemical Co. (St. Louis, USA) unless specified otherwise.

\subsection{Experimental design}

In this experiment, 1686 oocytes collected from 859 ovaries were used to produce ovine embryos that were subjected to a $2 \times 2$ factorial experimental design with two maturation media (M1, maturation medium usually used in our Lab as in Pereira et al. (2009), (836) immature oocytes; or M2, M1 plus FSH and Na pyruvate, 850 immature oocytes) and two semen treatments (fresh-F or frozen-thawed-FT). The three Merino rams used and sessions or batch of ovaries were considered random effects. Each ram was tested three times running once individually and twice simultaneously with each other, performing a total of 9 replicates. Morphological parameters as well as the capacitation status of fresh and frozen-thawed ram semen prior to IVF were evaluated. Cleavage was assessed by microscopy $48 \mathrm{~h}$ after fertilization $(\mathrm{IVF}=$ day 0$)$. On day 6 till day 8 , embryos were evaluated for developmental and morphological status (grade 1, good and grade 3, poor). Grade 1 embryos $(\mathrm{M} 1 \times \mathrm{FT}$, $n=19 ; \mathrm{M} 2 \times \mathrm{FT}, n=27 ; \mathrm{M} 1 \times \mathrm{F}, n=30 ; \mathrm{M} 2 \times \mathrm{F}, n=29$ ) were vitrified. After warming ( 3 sessions), embryo viability was evaluated by its integrity and re-expansion as well as after $3 \mathrm{~h}$ of in vitro culture. The number of cells (total and viable) in thawed/cultured blastocysts (2-6 embryo per treatment in each session) was also assessed.

\subsection{Oocyte in vitro maturation}

Ovaries collected from crossbred Merino sheep at a local slaughterhouse were transported to the laboratory in Dulbecco's phosphate buffer saline (PBS, GibCo $14040-91$ ) at $35^{\circ} \mathrm{C}$. PBS was supplemented with $0.15 \%$ $(\mathrm{w} / \mathrm{v})$ of bovine serum albumin (BSA) and $0.05 \mathrm{mg} \mathrm{mL}^{-1}$ of kanamycin. At the laboratory, the 2-6 $\mathrm{mm}$ follicles were aspirated to obtain the cumulusoocyte complexes (COC).

Selected COC were divided and incubated in two different maturation media (M1 and $\mathrm{M} 2$ ) at $38.5^{\circ} \mathrm{C}$ and $5 \% \mathrm{CO}_{2}$ for $22 \mathrm{~h}$. The composition of first medium (M1) included medium $199,10 \mu \mathrm{M}$ cysteamine, $10 \mathrm{ng} \mathrm{mL}^{-1} \mathrm{EGF}$, $10 \mu \mathrm{g} \mathrm{mL}^{-1}$ estradiol and $10 \mu \mathrm{L} \mathrm{mL}^{-1}$ gentamicin (Pereira et al., 2009). The second medium (M2) was also supplemented with $0.3 \mathrm{mM}$ sodium pyruvate and $10 \mu \mathrm{g} \mathrm{mL} \mathrm{m}^{-1} \mathrm{FSH}$.

The apparent maturation was evaluated (mature oocytes scored morphologically/immature oocytes). Oocytes were considered mature if a homogenous cytoplasm and multilayered, expanded cumulus cells were present.

\subsection{Semen collection}

Semen collection was conducted at the experimental farm of INRB in compliance with the requirements of the European Union for farm animal welfare and the Portuguese authority guidelines for animal experimentation.

Semen was collected from three Portuguese Merino rams of proven fertility by an artificial vagina. Each ejaculate was immediately evaluated for volume, motility and concentration. Only semen with good quality (mass motility $>4$; individual motility $>60 \%$; concentration $>2.5 \times 10^{9} \mathrm{spz} \mathrm{mL}^{-1}$ ) was used either fresh or cryopreserved.

\subsection{Semen cryopreservation}

Ejaculates with good quality were diluted in a solution of $45.0 \mathrm{~g} \mathrm{~L}^{-1}$ Tris, 24.4 $\mathrm{g} \mathrm{L}^{-1}$ citric acid (Merck 1.002.441.000), $5.6 \mathrm{~g} \mathrm{~L}^{-1}$ glucose, $15 \%$ egg yolk (v/v), 6.6\% glycerol (v/v, Merck 1.040.011.000) and antibiotics (Marques et al., 2006). After dilution, semen was packed in $0.25 \mathrm{~mL}$ Cassou straws $\left(300 \times 10^{6} \mathrm{spz}\right)$ and refrigerated $\left(4^{\circ} \mathrm{C}\right)$ for $4 \mathrm{~h}$ prior to freezing in liquid nitrogen $\left(\mathrm{LN}_{2}\right)$ vapor during $25 \mathrm{~min}$ and then submersed and kept in a $\mathrm{LN}_{2}$ container (Valente et al., 2010).

\subsection{Preparation and evaluation of fresh and thawed semen}

After collection, fresh ejaculates were kept at room temperature $\left(22^{\circ} \mathrm{C}\right)$ and light protected for up to $2 \mathrm{~h}$, then washed in synthetic oviductal fluid (SOF) and centrifuged at $225 \times \mathrm{g}$ for $5 \mathrm{~min}$. This medium was SOF enriched with bovine serum albumin $\left(4 \mathrm{mg} \mathrm{mL}^{-1}\right.$ BSA) plus glutamine $\left(1.5 \mu \mathrm{g} \mathrm{mL}^{-1}\right)$ and kept at the same room temperature (adapted from Dattena et al., 2000). Subsequently washed spermatozoa were diluted with $1 \mathrm{~mL}$ of fertilization medium consisting of SOF containing BME $\left(20 \mu \mathrm{LL}^{-1}\right)$ and MEM amino acids $\left(10 \mu \mathrm{LL}^{-1}\right)$, gentamicin $\left(10 \mu \mathrm{L} \mathrm{mL}^{-1}\right)$ and $10 \%$ ovine superovulated oestrus serum.

After thawing, sperm motility was immediately examined. Frozen-thawed semen was then incubated at $38.5^{\circ} \mathrm{C}$ and $5 \% \mathrm{CO}_{2}$ for $1 \mathrm{~h}$ in capacitation medium (modified Brackett's medium containing $20 \%$ ovine superovulated oestrus serum, Pereira et al., 2009). After centrifugation at $225 \times g$ for $5 \mathrm{~min}$, the supernatant were rejected and the 
remaining pellet of spermatozoa evaluated prior to be used to fertilize the oocytes in fertilization medium.

\subsubsection{Morphological parameters}

Either post swim-up thawed or washed fresh sperm were evaluated for individual motility (percentage of progressively motile spermatozoa), vigor (scale 0-5) and concentration.

\subsubsection{Capacitation}

The capacitation status of swimmed-up thawed or washed fresh sperm were assessed by chlortetracycline (CTC) staining binding pattern in the spermatozoa membranes using the technique described by Pereira et al. (2009). Aliquots ( $5 \mu \mathrm{L})$ of motile spermatozoa were mixed with $5 \mu \mathrm{L}$ of CTC solution $\left(0.4 \mathrm{~g} \mathrm{~L}^{-1} \mathrm{CTC}-\mathrm{HCl}, 0.9 \mathrm{~g} \mathrm{~L}^{-1}\right.$ cystein in $5 \mathrm{~mL}$ of $2.4 \mathrm{~g} \mathrm{~L}^{-1}$ Tris and $7.6 \mathrm{~g} \mathrm{~L}^{-1} \mathrm{NaCl}$ solution), $1 \mu \mathrm{L}$ of $12.5 \%$ glutaraldehyde solution and $1 \mu \mathrm{L}$ of DABCO (Merck 8.03456). Slides were observed under fluorescence within $12 \mathrm{~h}$ and spermatozoa classified according to their acrosomal status as: uncapacitated acrosome intact; capacitated acrosome intact; and acrosome reacted cells.

\subsection{In vitro fertilization and embryo culture}

Procedures were as described by Pereira et al. (2009). Briefly, sheep matured COC were co-cultured with fresh or thawed spermatozoa $\left(1 \times 10^{6} \mathrm{spz} \mathrm{mL}^{-1}\right)$ in SOF containing BME and MEM amino acids, gentamicin and $10 \%$ ovine superovulated oestrus serum as described above.

Eighteen hours after insemination, presumptive zygotes were transferred into $25 \mu \mathrm{L}$ droplets of SOF supplemented with BME and MEM amino acids and bovine serum albumin $\left(6 \mathrm{mg} \mathrm{mL}^{-1} \mathrm{BSA}\right)$ until the stage of $2-8$ cells. After assessing cleavage using a stereo microscope (Olympus SZ60), embryo development proceeded until the blastocyst stage in amino acids and BSA supplemented SOF plus 10\% fetal calf serum (SOFserum). Embryo culture was performed at $38.5^{\circ} \mathrm{C}$ in a humidified atmosphere with $5 \% \mathrm{O}_{2}, 5 \% \mathrm{CO}_{2}$ and $90 \% \mathrm{~N}_{2}$.

Cleavage rate was calculated as the number of cleaved embryos per number of inseminated oocytes. Day 6(D6) and D8 embryo developmental rates were calculated as the number of D6 or D8 blastocysts per number of cleaved embryos, respectively.

Embryo quality (D6-D8) was classified on the basis of conventional morphological criteria and according to their stage of development following the guidelines of the International Embryo Transfer Society (grade 1: good, no blemishes or only trivial imperfections; grade 2: fair, with some extruded or degenerated cells and non-uniform, darker appearance; and grade 3: bad, poor quality, lacking cohesion or with many extruded or degenerated cells).

\subsection{Blastocyst vitrification and warming}

A proportion of grade 1 blastocysts was vitrified using the open-pulled straw (OPS) method described by Vajta et al. (1998). Blastocysts were first equilibrated in holding medium (HM, M199+20\% FCS) for 5 min, then in diluted (HM with 10\% ethylene glycol-EG and 10\% dimethyl sulfoxideDMSO, $1 \mathrm{~min}$ ) and concentrated (HM with 20\% EG and 20\% DMSO) vitrification media. Embryos were quickly placed into the superfine OPS and plunged directly into $\mathrm{NL}_{2}(30 \mathrm{~s})$. Warming was performed by placing the end of the straw directly into the holding medium. After 2 min, blastocysts were transferred into HM for another $2 \mathrm{~min}$; and then to SOFserum and cultured for $3 \mathrm{~h}$. Samples of these embryos (2-6 embryo per treatment in each post-thawed session) were fixed and stained for cell number and viability evaluation.

Embryos integrity and re-expansion (Pereira et al., 2007) were assessed $20 \mathrm{~min}$ post-thawing and after $3 \mathrm{~h}$ of in vitro culture (Morató et al., 2011). Embryo integrity rate was calculated as the number of intact embryos (blastocysts with an intact zona pellucida and without several extruded blastomeres lacking cohesion) per number of frozen-thawed embryos. Embryo re-expansion rate was calculated as the number of reexpanded embryos (blastocysts that recovered their original shape with re-expanded blastocoels) per number of frozen-thawed embryos.

\subsection{Evaluation of embryo cell viability and total number}

After culture, post-thawed grade 1 blastocysts were washed in PBS, transferred into pronase containing medium for $1.5 \mathrm{~min}$ and then to
Tyrodes medium for another $1.5 \mathrm{~min}$. Afterwards the embryos without the zona pelucida were washed again in PBS and incubated in SOFserum for $1 \mathrm{~h}$ prior to fixation in a 4\% paraformaldehyde solution and staining in a solution of $50 \mu \mathrm{g} \mathrm{mL}^{-1}$ of Hoechst 33342 and $5 \mu \mathrm{g} \mathrm{mL}^{-1}$ of propidium iodide (PI) (adapted from Hosseini et al., 2007).

Finally embryos were individually placed into $2 \mu \mathrm{L}$ of Mowiol (Calbiochem 475904) medium on glass slides and covered with a coverslip. Slides were refrigerated and kept in dark until being observed in a widefield fluorescence microscope (Leica DM5000B) using red and ultraviolet excitation. Images were saved using the software Image J (National Institutes of Health, USA) and total cell number (Hoechst 33342 stain) and dead cells (PI stain) counted.

\subsection{Statistical analysis}

Procedure MIXED of Statistical Analysis Systems Institute (SAS Inst., Inc., Cary, NC, USA) was used to analyze data with a model including. embryo production (cleavage, D6 and D8 embryos rates) as well as the number of cells in each post-thawed embryo and their viability. Mixed linear model included in vitro maturation media and semen status as fixed effects and combination of ram*replicate as random effect. In addition the means for each treatment were calculated as well as the differences between the means and the respective $t$-test. Proc MIXED of SAS was also used to analyze data from apparent maturation and semen evaluation with a model including in vitro maturation media or semen status as fixed effects, respectively and in the latter case the combination ram*replicate as random effect.

Data from embryo quality (good, fair and bad) and frozen-thawed embryos survival were analyzed using GLIMMIX procedure from SAS with a model that included the effect of in vitro maturation media and semen status as fixed effects and ram as random effect.

Results were considered statistically different when $P \leq 0.05$

\section{Results}

The apparent maturation rates presented no differences $(P>0.05)$ between treatments $(M 1=87.0 \pm 4.1$ and $\mathrm{M} 2=86.7 \pm 3.9 \%)$. Equally, no differences were found among rams $(n=3)$ semen parameters prior to IVF. However, as expected, initial $(P<0.0001)$ and pre-IVF $(P=0.05)$ motility and vigor $(P=0.04)$ in fresh semen were superior to those of frozen-thawed semen (Table 1). The capacitation status of swimmed-up spermatozoa presented no differences $(P>0.05)$ between fresh and frozen-thawed semen.

Similar embryo production rates were obtained in all groups (Table 2), without significant effects of maturation media and semen status or interaction between them.

Grade 1 embryos rate using $F$ semen was higher $(P=0.01)$ than with FT semen $(54.6 \pm 7.7 \%$ and $39.5 \pm 7.3 \%$, respectively). Interestingly, there was no significant effect of the maturation media or interaction between maturation media and semen status on this parameter (Fig. 1). However this interaction was significant $(P=0.03)$ for grade 2 embryos being the rate of these blastocysts lower $(P=0.007)$ in $\mathrm{M} 2 \times \mathrm{F}(19.3 \pm 5.6 \%)$ group than in $\mathrm{M} 2 \times \mathrm{FT}$ $(40.0 \pm 5.5 \%)$. The maturation media and semen status did not influence $(P>0.05)$ on grade 3 blastocyst rate.

One hundred and five good quality embryos were vitrified and warmed to evaluate post-viability (Table 3). After warming, similar results were obtained in embryo integrity and re-expansion rates. However, after $3 \mathrm{~h}$ of in vitro culture, the expansion rate was higher in $\mathrm{M} 2 \times \mathrm{F}$ than in $\mathrm{M} 1 \times \mathrm{F}$. No differences were found in embryo total cells number or in their viability among treatments. 
Table 1

Evaluation of morphological parameters and capacitation status (chlortetracycline staining-CTC) of fresh (F) and frozen thawed (FT) ovine semen (least squares means \pm standard error).

\begin{tabular}{|c|c|c|c|c|c|c|c|}
\hline \multirow[t]{2}{*}{ Semen status } & \multirow[t]{2}{*}{$N$} & \multicolumn{3}{|c|}{ Morphological parameters } & \multicolumn{3}{|l|}{ CTC } \\
\hline & & Initial motility (\%) & Pre-IVF motility (\%) & Vigor $(0-5)$ & NCap (\%) & Cap (\%) & $\mathrm{AR}(\%)$ \\
\hline $\mathrm{F}$ & 9 & $75.2 \pm 1.8^{\mathrm{a}}$ & $77.4 \pm 3.5^{\mathrm{a}}$ & $4.7 \pm 0.1^{\mathrm{a}}$ & $39.2 \pm 3.4$ & $41.9 \pm 2.9$ & $19.8 \pm 2.8$ \\
\hline FT & 9 & $45.5 \pm 1.8^{b}$ & $66.3 \pm 3.5^{b}$ & $4.4 \pm 0.1^{\mathrm{b}}$ & $28.8 \pm 3.4$ & $47.0 \pm 2.9$ & $24.1 \pm 2.8$ \\
\hline
\end{tabular}

Data within the same columns with different superscripts letters are statistically different $(p \leq 0.05)$; NCap: uncapacitated acrosome intact cells; Cap: capacitated acrosome intact cells; AR: acrosome reacted cells; IVF: in vitro fertilization.

\section{Table 2}

Effect of different maturation media (M1 and M2) and oocyte fertilization by fresh (F) or frozen-thawed (FT) spermatozoa on embryo production rates (least squares means \pm standard error and $P$-values).

\begin{tabular}{lllll}
\hline Treatment & $N$ & Cleavage (\%) & D6 embryo (\%) & D8 embryo (\%) \\
\hline M1 $\times$ FT & 371 & $48.0 \pm 5.4$ & $46.9 \pm 3.8$ & $38.5 \pm 3.2$ \\
M2 $\times$ FT & 359 & $45.1 \pm 3.5$ & $44.5 \pm 3.0$ & $42.8 \pm 3.3$ \\
M1 $\times$ F & 353 & $44.2 \pm 4.7$ & $42.6 \pm 4.1$ & $37.3 \pm 5.3$ \\
M2 $\times$ F & 372 & $41.9 \pm 5.1$ & $43.0 \pm 3.7$ & $37.4 \pm 5.5$ \\
Total & 1455 & $44.8 \pm 2.3$ & $44.24 \pm 1.8$ & $39.0 \pm 2.2$ \\
& $\mathrm{M}$ & $P=0.358$ & $P=0.382$ & $P=0.414$ \\
Effects & $\mathrm{S}$ & $P=0.495$ & $P=0.762$ & $P=0.594$ \\
& $\mathrm{M} \times \mathrm{S}$ & $P=0.939$ & $P=0.666$ & $P=0.614$
\end{tabular}

M: maturation media; M1: maturation medium containing EGF; M2: maturation medium containing EGF, pyruvate and FSH; $N$ : inseminated oocytes; S: semen status.

\section{Discussion}

Although in vitro production and cryopreservation of ovine embryos can be an useful technique in reproductive biotechnology, the technical feasibility and quality of the produced embryos are lower than for other species (Thibier and Guérin, 2000; Pereira and Marques, 2008). Improvement of practical results could trigger a more extensive application for this technique. Our goal was to compare the use of two different maturation media and type of semen for IVF in order to optimize results. Data presented here demonstrated that fertilization of matured ovine oocytes with F semen improves the morphological quality of the produced embryos. Moreover an enhanced embryo cryosurvival is achieved when a maturation medium is supplemented with FSH, EGF and pyruvate, allied to the F semen used for IVF.

As referred, the individual effect of FSH/EGF or pyruvate on the cytoplasmatic maturation of the oocyte has been thoroughly investigated. Accordingly, FSH or EGF is largely used in many in vitro maturation protocols because it has been shown to improve cumulus expansion, oocyte cytoplasmatic maturation, fertilization and early embryonic development (Paria and Dey, 1990; Wang et al., 2009). Recently a synergistic effect of FSH and EGF on cytoplasmic maturation of porcine oocytes was reported by Uhm et al. (2010). Moreover we hypothesized that pyruvate could minimize the energy imbalance in the oocyte referred by Sugiura et al. (2005), due to the stimulation of HBP by FSH/EGF during in vitro maturation. Therefore by adding these components to the maturation media we tried to improve in vitro maturation of ovine oocytes through a synergistic effect of $\mathrm{FSH}$, EGF and pyruvate. Although no differences were identified in maturation, cleavage and D6-D8 rates, FSH, EGF and pyruvate presence improved the oocyte developmental competence. In fact, after $3 \mathrm{~h}$ of culture, expansion rate of vitrified-warmed grade 1 quality embryos in $\mathrm{M} 2 \times \mathrm{F}$ group was higher $(P=0.05)$ than in $\mathrm{M} 1 \times \mathrm{F}$ group. Thus, it seems that FSH, EGF and pyruvate contribute to regulate ovine oocyte maturation and optimize their developmental competence in vitro, namely when using F semen for IVF.

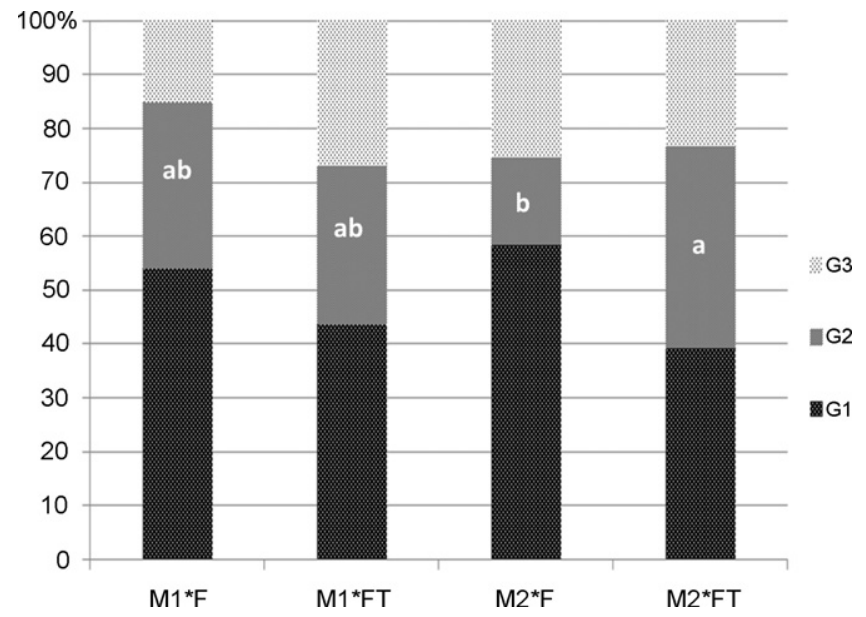

Fig. 1. Percentual distribution of embryo $(n=279)$ morphological quality [grade 1 embryos (G1); grade 2 embryos (G2); grade 3 embryos (G3)] in the four groups according to maturation method (M1: maturation medium containing EGF and M2: medium containing EGF, pyruvate and FSH) and type of semen used: fresh (F) or frozen-thawed (FT) $(\mathrm{a} \neq \mathrm{b}, P=0.007)$. 
Table 3

Effect of different maturation media (M1 and M2) and oocyte fertilization by fresh (F) or frozen-thawed (FT) spermatozoa on post-thawed embryo ( $n=105$ ) viability evaluated by intact and re-expanded blastocyst rates and embryo cell counts (least squares means \pm standard error).

\begin{tabular}{|c|c|c|c|c|c|c|}
\hline \multicolumn{2}{|c|}{ Treatments } & \multicolumn{3}{|c|}{ Post-thawed } & \multicolumn{2}{|l|}{ After culture } \\
\hline & & $N$ & Intact embryos (\%) & Re-expanded embryos (\%) & Re-expanded embryos (\%) & Total cells (viability) \\
\hline M1 & FT & 30 & $80.7 \pm 8.3$ & $77.4 \pm 6.9$ & $74.7 \pm 8.9^{\mathrm{ab}}$ & $56.6 \pm 8.2(33.5 \%)$ \\
\hline M2 & FT & 19 & $69.4 \pm 11.9$ & $65.3 \pm 15.3$ & $60.0 \pm 12.0^{\mathrm{ab}}$ & $59.4 \pm 11.5(20.3 \%)$ \\
\hline M1 & $\mathrm{F}$ & 29 & $76.2 \pm 9.3$ & $71.8 \pm 15.5$ & $54.3 \pm 10.4^{\mathrm{b}}$ & $65.0 \pm 8.6(36.6 \%)$ \\
\hline M2 & $\mathrm{F}$ & 27 & $73.2 \pm 15.5$ & $69.0 \pm 10.1$ & $80.0 \pm 8.3^{a}$ & $48.9 \pm 12.8(39.2 \%)$ \\
\hline
\end{tabular}

Data within the same columns with different superscripts letters are statistically different $(P \leq 0.05)$; M1: maturation medium containing EGF; M2: maturation medium containing EGF, pyruvate and FSH; $N$ : inseminated oocytes.

Cryopreservation severely damages ram spermatozoa. Therefore as expected and previously documented (Bailey et al., 2000; Marques et al., 2006), the classical andrological sperm parameters were reduced in quality following cryopreservation. The observation that cryopreserved ram spermatozoa have more susceptible and heterogeneous chromatin than fresh, suggests that the poorer fertilizing efficiency of frozen ram semen might be at least partly due to abnormal sperm DNA structure, despite having a normal appearance soon after thawing (Peris et al., 2004). Interestingly similar cleavage, D6 and D8 embryo rates were obtained independently of the semen status, F or postthawed, in the present study. In accordance Lehloenya et al. (2010) also reported no significant differences between $\mathrm{F}$ and FT semen regarding the embryonic developmental stages although presenting a blastocyst rate ranging between $0.4 \pm 0.4 \%$ and $2.6 \pm 1.0 \%$, respectively.

Despite some previous results that had found no differences in the cleavage rates between oocytes inseminated with F or FT semen (Pugh et al., 1991; Lehloenya et al., 2010), present results demonstrate that the use $F$ sperm for in vitro fertilization can improve ovine embryo developmental competence by enhancing morphological blastocyst quality. The inferior viability of in vitro produced embryos is attributed to cellular (Pereira et al., 2007; Rizos et al., 2002), metabolic (Khurana and Niemann, 2000; Farin et al., 2001) and biochemical changes (Massip et al., 1995). In vitro ovine blastocysts exhibited less microvilli and a less extensive network of intercellular junctions, specifically an apparent lack of desmosomal junctions, a higher incidence of cellular debris and a higher number of lipid droplet than their in vivo counterparts (Farin et al., 2001; Rizos et al., 2002). These differences could certainly contribute to the observed lower cryotolerance of in vitro produced embryos.

Embryo morphology and post-thawing viability have been widely used to determine embryo viability (Dattena et al., 2000, 2004; Pereira et al., 2007). In addition to the above mentioned beneficial effects of FSH, EGF and pyruvate on in vitro maturation, FSH promotes the synthesis of hyaluronic acid (HA) in expanding cumulus cells during maturation. Moreover, HA synthesis during cumulus mucification contributes to the penetration and fertilization of oocytes, most likely facilitating the process of capacitation and acrosome reaction and subsequent developmental competence (Gutnisky et al., 2007). This could explain that herein besides the improvement of blastocyst quality when using $\mathrm{F}$ semen for IVF, an interaction between semen status and maturation media on embryo quality and cryosurvival was identified. Whereas $\mathrm{M} 2 \times \mathrm{F}$ group presented less grade 2 embryos than M $2 \times$ FT group, the expansion rate of vitrified-warmed grade 1 embryos from $\mathrm{M} 2 \times \mathrm{F}$ group was higher than in $\mathrm{M} 1 \times \mathrm{F}$ group. When using FT semen, unexpectedly no differences were identified in embryo post-thawed expansion rates. Thus the association of a maturation medium containing FSH, EGF and pyruvate with F semen for IVF should be used to enhance the viability of ovine in vitro produced embryos.

\section{Conclusion}

In conclusion presented results seem to confirm the existence of a synergistic effect between FSH, EGF and pyruvate on cytoplasmic maturation of ovine oocytes. In addition, in vitro fertilization by fresh semen clearly improves ovine embryo developmental competence by enhancing morphological blastocyst quality. The beneficial effect of M2 on cryosurvival was only observed in embryos derived from fresh semen. Therefore these combined strategies enhance embryo cryosurvival.

\section{Acknowledgements}

This work was supported by funds from the Portuguese Foundation for Science and Technology (PPTDC/ CVT/98607/2008) and PhD Grant (SFRH/BD/37853/2007).

\section{References}

Bailey, J.L., Bilodeau, J.F., Cormier, N., 2000. Semen cryopreservation in domestic animals: a damaging and capacitating phenomenon. J. Androl. 21, 1-7.

Buccione, R., Vanderhyden, B.C., Caron, P.J., Eppig, J.J., 1990. FSH-induced expansion of the mouse cumulus oophorus in vitro is dependent upon a specific factor(s) secreted by the oocyte. Dev. Biol. 138, 16-25.

Cognié, Y., Baril, G., Poulin, N., Mermillod, P., 2003. Current status of embryo technologies in sheep and goat. Theriogenology 59, 171-188.

Dattena, M., Accardo, C., Pilchi, S., Isachenko, V., Mara, L., Chessa, B., Cappai, P., 2004. Comparison of different vitrification protocols on viability after transfer of ovine blastocysts in vitro produced and in vivo derived. Theriogenology 62, 481-493.

Dattena, M., Ptak, G., Loi, P., Cappai, P., 2000. Survival and viability of vitrified in vitro and in vivo produced ovine blastocysts. Theriogenology 53, 1511-1519.

Farin, C.E., Rodriguez, K.F., Alexander, J.E., Hockney, J.E., Herrick, J.R., Kennedy-Stoskopf, S., 2007. The role of transcription in EGF- and FSH-mediated oocyte maturation in vitro. Anim. Reprod. Sci. 98, 97-112.

Farin, P.W., Crosier, A.E., Farin, C.E., 2001. Influence of in vitro systems on embryo survival and fetal development in cattle. Theriogenology 55, 151-170. 
Feng, P., Knecht, M., Catt, K.J., 1987. Hormonal control of epidermal growth factor receptors by gonadotronins during granulosa cell differentiation. Endocrinology 120, 1121-1126.

Guler, A., Poulin, N., Mermillod, P., Terqui, M., Cognié, Y., 2000. Effect of growth factors, EGF and IGF-1, and estradiol on in vitro maturation of sheep oocytes. Theriogenology 54, 209-218.

Gutnisky, C., Dalvit, G.C., Pintos, L.N., Thompson, J.G., Beconi, M.T., Cetica, P.D., 2007. Influence of hyaluronic acid synthesis and cumulus mucification on bovine oocyte in vitro maturation, fertilisation and embryo development. Reprod. Fertil. Dev. 19, 488-497.

Hosseini, S.M., Hajian, M., Asgari, V., Forozanfar, M., Abedi, P., NasrEsfahani, M.H., 2007. Novel approach of differential staining to detect necrotic cells in preimplantation embryos. Iran. J. Fertil. Steril. 1, 103-106.

Kelly, J.M., Kleemann, D.O., Maxwell, W.M.C., Walker, S.K., 2008. Effects of insuline-like growth factor-I, epidermal growth factor and cysteamine on the in vitro maturation and development of oocytes collected from 6- to 8-week-old Merino lambs. Reprod. Fertil. Dev. 20, 570-578.

Khurana, N.K., Niemann, H., 2000. Effects of cryopreservation on glucose metabolism and survival of bovine morulae and blastocyst derived in vitro or in vivo. Theriogenology $54,313-326$.

Lehloenya, K.C., Mahoete, N., Greyling, J.P.C., Nedambale, T.L., 2010. Effect of breed and frozen-thawed ram semen on in vitro fertilization and ovine embryonic development. Reprod. Fertil. Dev. 23, 170.

Massip, A., Mermillod, P., Dinnyes, A., 1995. Morphology and biochemistry of in vitro produced bovine embryos: implications for their cryopreservation. Hum. Reprod. 10, 3004-3011.

Marques, C.C., Barbas, J.P., Baptista, M.C., Cannas-Serra, C., Vasques, M.I., Pereira, R.M., Cavaco-Gonçalves, S., Horta, A.E.M., 2006. Reproduction in the ovine Saloia breed: seasonal and individual factors affecting fresh and frozen semen performance, in vivo and in vitro fertility. In: Ramalho Ribeiro, J.M.C., Horta, A.E.M., Mosconi, C., Rosati, A. (Eds.), Animal Products from the Mediterranean Area - EAAP 119. Wageningen Academic Publishers, The Netherlands, pp. 331-336.

Morató, R., Romaguera, R., Izquierdo, D., Paramio, M.T., Mogas, T., 2011. Vitrification of in vitro produced goat blastocysts: effects of oocyte donor age and developmental stage. Cryobiology 63, $240-244$.

Papadopoulos, S., Rizos, D., Duffy, P., Wade, M., Quinn, K., Boland, M.P., Lonergan, P., 2002. Embryo survival and recipient pregnancy rates after transfer of fresh or vitrified, in vivo or in vitro produced ovine embryos. Anim. Reprod. Sci. 74, 35-44.

Paria, B.C., Dey, S.K., 1990. Preimplantation embryo development in vitro: cooperative interactions among embryos and role of growth factors. Proc. Natl. Acad. Sci. U. S. A. 80, 4756-4760.

Pereira, R.M., Mesquita, P., Batista, M., Baptista, M.C., Barbas, J.P., Pimenta, J., Santos, I.C., Marques, M.R., Vasques, M.I., Silva Pereira, M., Santos Silva, F., Oliveira Sousa, M.C., Fontes, C.M., Horta, A.E.M., Prates, J.A., Marques, C.C., 2009. Doppel gene polymorphisms in Portuguese sheep breeds: insights on ram fertility. Anim. Reprod. Sci. 114, 157-166.
Pereira, R.M., Baptista, M.C., Vasques, M.I., Horta, A.E.M., Portugal, P.V., Bessa, R.J.B., Chagas e Silva, J., Silva Pereira, M., Marques, C.C., 2007. Cryo-survival of bovine blastocysts is enhanced by culture with trans10 cis-12 conjugated linoleic acid (10t, 12c CLA). Anim. Reprod. Sci. 98, 293-301.

Pereira, R.M., Marques, C.C., 2008. Animal oocyte and embryo cryopreservation. Cell Tissue Bank 9, 267-277.

Peris, S.I., Morrier, A., Dufour, M., Bailey, J.L., 2004. Cryopreservation of ram semen facilitates sperm DNA damage: relationship between sperm andrological parameters and the sperm chromatin structure assay. J. Androl. 25, 224-233.

Procházka, R., Petlach, M., Nagyová, E., Nemcová, L., 2011. Effect of epidermal growth factor-like peptides on pig cumulus cell expansion, oocyte maturation, and acquisition of developmental competence in vitro: comparison with gonadotropins. Reproduction 141, 425-435.

Pugh, P.A., Fukui, Y., Tervit, H.R., Thompson, J.G., 1991. Developmental ability of in vitro matured sheep oocytes collected during the nonbreeding season and fertilized in vitro with frozen ram semen. Theriogenology 36, 771-778.

Rizos, D., Ward, F., Duffy, P., Boland, M.P., Lonergan, P., 2002. Consequences of bovine oocyte maturation, fertilization or early embryo development in vitro versus in vivo: implications for blastocyst yield and blastocyst quality. Mol. Reprod. Dev. 61, 234-248.

Sugiura, K., Pendola, F.L., Eppig, J.J., 2005. Oocyte control of metabolic cooperativity between oocytes and companion granulosa cells: energy metabolism. Dev. Biol. 279, 20-30.

Thibier, M., Guérin, B., 2000. Embryo transfer in small ruminants: the method of choice for health control in germplasm exchanges. Livest. Prod. Sci. 62, 253-270.

Uhm, S.J., Gupta, M.K., Yang, J.H., Chung, H.-J., Min, T.S., Lee, H.T., 2010. Epidermal growth factor can be used in lieu of follicle-stimulating hormone for nuclear maturation of porcine oocytes in vitro. Theriogenology 73, 1024-1036.

Valente, S., Pereira, R.M., Baptista, M.C., Marques, C.C., Vasques, M.I., Silva Pereira, M.V.C., Horta,.A.E.M., Barbas, J.P., 2010. In vitro and in vivo fertility of ram semen cryopreserved in different extenders. Anim. Reprod. Sci. 117, 74-77.

van de Leemput, E.E., Vos, P.L., Zeinstra, E.C., Bevers, M.M., van der Weijden, G.C., Dieleman, S.J., 1999. Improved in vitro embryo development using in vivo matured oocytes from heifers superovulated with a controlled preovulatory LH surge. Theriogenology 52 , 335-349.

Vajta, G., Holm, P., Kuwayama, M., Booth, P.J., Jacobsen, H., Greve, T., Callesen, H., 1998. Open pulled straws (OPS) vitrification: a new way to reduce cryoinjuries of bovine ova and embryos. Mol. Reprod. Dev. 51, 53-58.

Wang, L.M., Feng, H.L., Ma, Y.Zh., Cang, M., Li, H.J., Yan, Zh., Zhou, P., Wen, J.X., Bou, S., Liu, D.J., 2009. Expression of IGF receptors and its ligands in bovine oocytes and preimplantation embryos. Anim. Reprod. Sci. 114, 99-108. 